\title{
A STOCHASTIC PARAMETER OPERATOR SYSTEM
}

\author{
G. Butsan and M. Kozachenko
}

Let $H$ be a real separable Hilbert space with the norm $|\cdot|_{1}, X(H)$ the Banach ring of all linear bounded operators on $H$ with the uniform operator norm $|\cdot|_{2}, \sigma_{2}(H)$ the ideal of all Hilbert-Schmidt operators in $X(H)$ with the Hilbert-Schmidt norm $|\cdot|_{3}$. Further let $\sigma_{2}(H)+I=G_{2}(H)$ be the topological semigroup with the topology transferred from $\sigma_{2}(H)$ by the shift of the identity operator $I,(\Omega, F, P)$ some probability space, $\sigma_{2}(H, \Omega)$ the Hilbert space of all random elements $\xi: \Omega \rightarrow \sigma_{2}(H)$ with the norms $|\cdot|_{4},|\xi|_{4}=\mathbf{E}|\xi|_{3}^{2}$ (and $|\xi|_{5}^{2}=$ $\left.\sup _{|x| \leq 1} \mathbf{E}|\xi x|_{1}^{2}\right), G_{2}(H, \Omega)=\sigma_{2}(H, \Omega)+I$ with corresponding topology, $\sigma_{s}^{t}$ a filtration of $\sigma$-algebras from $F$ when $0 \leq s \leq t \leq T \leq \infty$, and for $0 \leq s \leq r \leq \tau \leq$ $t \leq T \leq \infty$, we have $\sigma_{r}^{\tau} \subset \sigma_{s}^{t}, \sigma_{s}^{r}$ and $\sigma_{\tau}^{t}$ are independent. Denote by $\Delta_{n}[s, t]$ the partition $\left\{s=t_{0} \leq t_{1} \leq \cdots \leq t_{m_{n}}=t\right\}$ of the interval $[s, t]$ and call the sequence $\left\{\Delta_{n}[s, t]\right\}$ a decreasing sequence of partitions if $\delta_{n}=\max _{k}\left(t_{k}-t_{k-1}\right) \rightarrow 0, n \rightarrow \infty$.

Now we consider a system $Z_{s}^{t}$ in $G_{2}(H, \Omega), 0 \leq s \leq t \leq T$, which is $\sigma_{s}^{t}$-measurable, and study the problem: when does the following limit exist in $G_{2}(H, \Omega)$

$$
\lim _{\delta_{n} \rightarrow 0} \prod_{k=1}^{m_{n}} Z_{t_{k-1}}^{t_{k}}=X_{s}^{t}
$$

and does not depend on the decreasing sequence of partitions $\left\{\Delta_{n}[s, t]\right\}$ ? Here the product $\Pi$ is formed in increasing order of the indices from left to right.

One of the problems is the existence of the infinite product of independent stochastic operators.

It is easy to see that $X_{s}^{t}$ satisfies the following evolutionary equation

$$
X_{s}^{\tau} X_{\tau}^{t}=X_{s}^{t}, \quad X_{\tau}^{\tau}=I(\bmod P), \quad 0 \leq s \leq \tau \leq t \leq T
$$

and therefore it is the evolutionary system from [1].

Denote

$$
\begin{aligned}
& z_{s}^{t}=\mathrm{E} Z_{s}^{t}, \quad \Delta_{n}[0, s]=\left\{0=\theta_{0} \leq \theta_{1} \leq \cdots \leq \theta_{p_{n}}=s\right\} \\
& \delta_{n}=\max _{i, k}\left\{\theta_{i}-\theta_{i-1}, t_{k}-t_{k-1}\right\}, \quad x_{0}^{t}(n)=\prod_{i=1}^{p_{n}} z_{\theta_{i-1}}^{\theta_{i}} \prod_{k=1}^{m_{n}} z_{t_{k-1}}^{t_{k}}, \\
& x_{s}^{t}(n)=\prod_{k=1}^{m_{n}} z_{t_{k-1}}^{t_{k}} .
\end{aligned}
$$


Theorem 1. If the system $Z_{s}^{t}$ satisfies the conditions (4)-(6), then the limit (1) exists in $G_{2}(H, \Omega)$ and does not depend on the decreasing sequence of partitions $\left\{\Delta_{n}[s, t]\right\}$. The conditions (4)-(6) are introduced as follows:

(4) for all $\tau \in[0, T]$, there exist $Z_{\tau-}^{\tau}, Z_{\tau}^{\tau^{+}} \in G_{2}(H, \Omega), Z_{\tau}^{\tau}=I$ satisfying $Z_{\tau-}^{\tau}=I$ or $Z_{\tau}^{\tau+}=I$,

(5) for all $0 \leq s \leq t \leq T$, there exists $\lim x_{s}^{t}(n)=x_{s}^{t} \in G_{2}(H)$ as $\delta_{n} \rightarrow 0$, $x_{s}^{t}$ does not depend on the decreasing sequence of partitions $\left\{\Delta_{n}[s, t]\right\}$, and $\gamma_{n}=$ $\sup \left|x_{0}^{t_{k}}-x_{0}^{t_{k}}(n)\right|_{2} \rightarrow 0$ as $\delta_{n} \rightarrow 0$; for all $\tau \in[0, T],\left(z_{\tau-}^{\tau}\right)^{-1},\left(z_{\tau}^{\tau+}\right)^{-1}, x_{\tau-}^{\tau}$, $x_{\tau}^{\tau+},\left(x_{\tau-}^{\tau}\right)^{-1},\left(x_{\tau}^{\tau+}\right)^{-1} \in X(H)$,

(6) there exists $\lim \sum\left(Z_{t_{k-1}}^{t_{k}}-z_{t_{k-1}}^{t_{k}}\right)=\check{Y}_{s}^{t} \in \sigma_{2}(H, \Omega)$ which does not depend on the decreasing sequence of partitions $\left\{\Delta_{n}[s, t]\right\}$.

Let us prove first some auxiliary equations (7)-(11), (12), (16), (17). We first claim that

$$
\text { for all } \tau \in[0, T], x_{\tau-}^{\tau}=z_{\tau-}^{\tau} \text { and } x_{\tau}^{\tau+}=z_{\tau}^{\tau+} \text {. }
$$

To prove (7) let us consider $\tilde{x}_{s}^{t}=\lim \prod_{k=1}^{m_{n}-1} z_{t_{k-1}}^{t_{k}}$. It follows from (5) that this limit exists and does not depend on the decreasing sequence $\left\{\Delta_{n}[s, t]\right\}$. Then it is easy to see that $x_{s}^{t}$ satisfies the evolutionary equation (2) and, by (5), that the $\operatorname{limit} \lim x_{s}^{\tau}=x_{s}^{t-}, \tau \uparrow t$, exists for $0 \leq s \leq t \leq T$.

Let us show that $x_{s}^{t-}=\tilde{x}_{s}^{t}$. In fact, when $\tau_{k} \uparrow t$, there exists, for any $\varepsilon>0, k_{\varepsilon}$ such that $\left|x_{s}^{t-}-x_{s}^{\tau_{k_{\varepsilon}}}\right|_{2}<\varepsilon / 2$. Thus, there exists $\Delta_{n_{k_{\varepsilon}}}\left[s, \tau_{k_{\varepsilon}}\right]=$ $\left\{s=\hat{t}_{0} \leq \cdots \leq \hat{t}_{r_{m}}=\tau_{k_{\varepsilon}}\right\}$ such that $\left|x_{s}^{\tau_{k_{\varepsilon}}}-\prod_{i=1}^{r_{m}} z_{\hat{t}_{i-1}}^{\hat{t}_{i}}\right|_{2}<\varepsilon / 2$. Therefore $\left|x_{s}^{t-}-\prod_{i=1}^{r_{m}} z_{\hat{t}_{i-1}}^{\hat{t}_{i}}\right|_{2}<\varepsilon$, and in this inequality we can take the limit when $\delta_{n_{k_{\varepsilon}}} \rightarrow 0$ because $\tilde{x}_{s}^{t}$ does not depend on the sequence of partitions $\left\{\Delta_{n}[s, t]\right\}$ (cf. (5)). Thus $x_{s}^{t-}=\tilde{x}_{s}^{t}$. Now using the corresponding limit procedure we can obtain the equality $x_{s}^{t-} z_{t-}^{t}=\tilde{x}_{s}^{t} z_{t-}^{t}=x_{s}^{t}$ yielding $x_{s}^{t-} z_{t-}^{t}=x_{s}^{t-} x_{t-}^{t}$. Taking the limit when $s \uparrow t$ and taking into account the evident equality $x_{t-}^{t-}=I$ (cf. (5)) we obtain the first equality in (7). The second equality can be proved analogously.

We show next that

$$
\begin{aligned}
& \text { for all } 0 \leq s \leq t \leq T,\left(x_{s}^{t}\right)^{-1} \in X(H),\left|x_{s}^{t}\right|_{2}<C_{1}(T)<\infty \\
& \left|\left(x_{s}^{t}\right)^{-1}\right|_{2}<C_{2}(T)
\end{aligned}
$$

If the first property of (8) is not fulfilled, then the statements $\left(x_{s}^{(t+s) / 2}\right)^{-1} \in X(H)$ or $\left(x_{(t+s) / 2}^{t}\right)^{-1} \in X(H)$, etc. would not be fulfilled either and we would obtain sequences $s_{k}<t_{k}$, converging to a point $\tau \in[0, T]$, such that $\left(x_{s_{k}}^{t_{k}}\right)^{-1} \notin X(H)$. Observe that we have three possibilities for these sequences: $s_{k} \leq t_{k} \leq \tau, s_{k} \leq$ $\tau \leq t_{k}$ or $\tau \leq s_{k} \leq t_{k}$. The first and third of them lead to a contradiction with the obvious equality $x_{\tau-}^{\tau-}=I=x_{\tau+}^{\tau+}(\mathrm{cf}$. (5)), whereas the second one contradicts with the equality $\left(x_{\tau-}^{\tau+}\right)^{-1}=\left(x_{\tau}^{\tau+}\right)^{-1} \cdot\left(x_{\tau-}^{\tau}\right)^{-1}$. 
Let us then prove the third inequality in (8) because the second one can be proved analogously. Let us suppose that $\left|\left(x_{s}^{t}\right)^{-1}\right|_{2}$ is not bounded. Then we can find in $[0, T]$ sequences $s_{k} \leq t_{k}$ converging to a point $\tau \in[0, T]$, and $\left|\left(x_{s_{k}}^{t_{k}}\right)^{-1}\right|_{2} \rightarrow \infty$ for $k \rightarrow \infty$. For all of the three cases considered above for analogous consequences, we obtain analogous contradictions with the equalities mentioned above.

Next we show that

$$
\sup _{k, n}\left|x_{0}^{t_{k}}(n)\right|_{2}<C_{3}(T)<\infty, \quad \sup _{k, n}\left|\left(x_{0}^{t_{k}}(n)\right)^{-1}\right|_{2}<C_{4}(T)<\infty .
$$

To prove the first one of these estimates consider the inequality

$$
\sup _{k, n}\left|x_{0}^{t_{k}}(n)\right|_{2} \leq \sup _{k, n}\left|x_{0}^{t_{k}}-x_{0}^{t_{k}}(n)\right|_{2}+\sup _{k}\left|x_{0}^{t_{k}}\right|_{2},
$$

which combined with (8) and the regularity conditions (5) completes our proof.

Let us suppose next that $\sup _{k, n}\left|\left(x_{0}^{t_{k}}(n)\right)^{-1}\right|_{2}=\infty$. Then we can find in $[0, T]$ a sequence $t_{k_{n}}$, which converges to a point $\tau \in[0, T]$ when $n \rightarrow \infty$ and $\left|\left(x_{0}^{t_{k_{n}}}(n)\right)^{-1}\right|_{2} \rightarrow \infty$ for $n \rightarrow \infty$. Then $\inf _{|y|_{1} \leq 1}\left|x_{0}^{t_{k_{n}}}(n) y\right|_{1} \rightarrow 0$ for $n \rightarrow \infty$, and from the evident inequality inf $\operatorname{iy|}_{1 \leq 1}\left|x_{0}^{t_{k_{n}}} y\right|_{1} \leq\left|x_{0}^{t_{k_{n}}}-x_{0}^{t_{k_{n}}}(n)\right|_{2}+\inf _{|y|_{1} \leq 1}\left|x_{0}^{t_{k_{n}}}(n) y\right|_{1}$ (cf. (5)) we obtain the result inf $\operatorname{in|}_{1} \leq 1\left|x_{0}^{t_{k_{n}}} y\right|_{1} \rightarrow 0$ when $n \rightarrow \infty$, i.e. $\left|\left(x_{0}^{t_{k_{n}}}\right)^{-1}\right|_{2} \rightarrow$ $\infty$. But this contradicts (8). Thus the estimates (9) are proved.

Now from the condition (2), estimates (8), (9) and the obvious inequality

$$
\sup _{k, n}\left|\left(x_{0}^{t_{k}}\right)^{-1}-\left(x_{0}^{t_{k}}(n)\right)^{-1}\right|_{2} \leq \sup _{k, n}\left\{\left|\left(x_{0}^{t_{k}}\right)^{-1}\right|_{2},\left|\left(x_{0}^{t_{k_{n}}}(n)\right)^{-1}\right|_{2}\right\} \sup _{k}\left|x_{0}^{t_{k}}-x_{0}^{t_{k}}(n)\right|_{2}
$$

we can see that

$$
\sup _{k, n}\left|\left(x_{0}^{t_{k}}\right)^{-1}-\left(x_{0}^{t_{k}}(n)\right)^{-1}\right|_{2} \rightarrow 0 \quad \text { when } n \rightarrow \infty .
$$

Let us show that the next equalities are true for all $\tau \in[0, T]$,

$$
\check{Y}_{\tau-}^{\tau}=Z_{\tau-}^{\tau-}-z_{\tau-}^{\tau}, \quad \check{Y}_{\tau}^{\tau+}=Z_{\tau}^{\tau+}-z_{\tau}^{\tau+} \quad(\bmod P) .
$$

As the proofs of these equalities are similar we prove only the first one. Let us consider the system

$$
\tilde{Y}_{s}^{t}=\lim _{\delta_{n} \rightarrow 0} \sum_{k=1}^{m_{n}-1}\left(Z_{t_{k-1}}^{t_{k}}-z_{t_{k-1}}^{t_{k}}\right) .
$$

The definition of $\tilde{Y}_{s}^{t}$ does not depend on the sequence $\left\{\Delta_{n}[s, t]\right\}$ (cf. (4), (6)), and analogously to (7) we can prove that $\tilde{Y}_{\tau-}^{\tau}=0$ for all $\tau \in[0, T]$. Taking the 
limit in the obvious equality $\check{Y}_{s}^{t}=Z_{t-}^{t}+z_{t-}^{t}+\tilde{Y}_{s}^{t}$ when $s \uparrow t$ we obtain the proof of the first equality (11).

Finally, from (4), (5), (11) we obtain:

$$
\begin{aligned}
& \text { for all } \tau \in[0, T], Z_{\tau-}^{\tau}=I, \quad z_{\tau-}^{\tau}=I, \quad x_{\tau-}^{\tau}=I, \quad \check{Y}_{\tau-}^{\tau}=0, \\
& \text { or } Z_{\tau}^{\tau+}=I, \quad z_{\tau}^{\tau+}=I, \quad x_{\tau}^{\tau+}=I, \quad \check{Y}_{\tau}^{\tau+}=0 \quad(\bmod P)
\end{aligned}
$$

In other words at any point $\tau \in[0, T]$ these systems are simultaneously continuous either from the left or from the right.

Let us note now that $\check{Y}_{s}^{t}$ (cf. (6)) satisfies the evolutionary equation

$$
\check{Y}_{s}^{\tau}+\check{Y}_{\tau}^{t}=\check{Y}_{s}^{t}, \quad \check{Y}_{\tau}^{\tau}=0, \quad(\bmod P), \quad 0 \leq s \leq t \leq T \leq \infty
$$

and the obvious property

$$
\mathbf{E} \check{Y}_{s}^{t}=0, \quad 0 \leq s \leq t \leq T \leq \infty
$$

which we used repeatedly in the considerations above. In addition, the random elements

$$
\check{Y}_{t_{k-1}}^{t_{k}} \quad, k=1, \ldots, m, \quad \text { are independent }
$$

for $0 \leq s=t_{0} \leq t_{1} \leq \cdots \leq t_{m_{n}}=T$.

It is easy to see that every system $\bar{Y}_{s}^{t}$ which satisfies the conditions (13)-(15) above satisfies the next relation too:

$$
\prod_{i=p}^{r}\left|\bar{Y}_{t_{i-1}}^{t_{i}}+I\right|_{5}^{2} \leq C_{5}(T)<\infty
$$

In fact, if we use (14) and the properties of $|\cdot|_{5}$ (cf. [1], pp. 48-52) we obtain the inequality

$$
\begin{aligned}
& \prod_{i=p}^{r}\left|I+\bar{Y}_{t_{i-1}}^{t_{i}}\right|_{5}^{2} \leq \prod_{i=p}^{r}\left(1+\left|\bar{Y}_{t_{i-1}}^{t_{i}}\right|_{5}^{2}\right) \leq \exp \left\{\sum_{i=p}^{r}\left|\bar{Y}_{t_{i-1}}^{t_{i}}\right|_{5}^{2}\right\} \\
& \leq \exp \left\{\sum_{i=p}^{r}\left|\bar{Y}_{t_{i-1}}^{t_{i}}\right|_{4}^{2}\right\} \leq \exp \left\{\left|\bar{Y}_{0}^{T}\right|_{4}^{2}\right\}=C_{5}(T)<\infty
\end{aligned}
$$

Let us then prove the following estimate

$$
\prod_{i=p}^{r}\left|x_{0}^{t_{i-1}}(n) Z_{t_{i-1}}^{t_{i}}\left(x_{0}^{t_{i}}(n)\right)^{-1}\right|_{5} \leq C_{6}(T)<\infty .
$$


For that purpose, let us apply the known properties of the norms $|\cdot|_{4}$ and $|\cdot|_{5}$ (cf. [1], pp. 48-52) and the estimates (9) to obtain the inequalities

$$
\begin{aligned}
& \prod_{i=p}^{r}\left|x_{0}^{t_{i-1}}(n) Z_{t_{i-1}}^{t_{i}}\left(x_{0}^{t_{i}}(n)\right)^{-1}\right|_{5}^{2} \\
& =\prod_{i=p}^{r}\left|x_{0}^{t_{i-1}}(n)\left(Z_{t_{i-1}}^{t_{i}}-z_{t_{i-1}}^{t_{i}}\right)\left(x_{0}^{t_{i}}(n)\right)^{-1}+I\right|_{5}^{2} \\
& \leq \exp \left(2\left\{\sum_{i=p}^{r}\left|x_{0}^{t_{i-1}}(n)\left(Z_{t_{i-1}}^{t_{i}}-z_{t_{i-1}}^{t_{i}}\right)\left(x_{0}^{t_{i}}(n)\right)^{-1}\right|_{4}^{2}\right\}\right) \\
& \leq \exp \left\{C_{3}^{2}(T) C_{4}^{2}(T) \sum_{i=p}^{r}\left|Z_{t_{i-1}}^{t_{i}}-z_{t_{i-1}}^{t_{i}}\right|_{4}^{2}\right\} \\
& \leq C_{7}(T) \exp \left\{\sum_{i=p}^{r}\left|Z_{t_{i-1}}^{t_{i}}-z_{t_{i-1}}^{t_{i}}-\check{Y}_{t_{i-1}}^{t_{i}}\right|_{4}^{2}+\left|\check{Y}_{t_{i-1}}^{t_{i}}\right|_{4}^{2}\right\} \\
& \leq C_{7}(T) \exp \left\{\sum_{i=1}^{m_{n}}\left|Z_{t_{i-1}}^{t_{i}}-z_{t_{i-1}}^{t_{i}}-\check{Y}_{t_{i-1}}^{t_{i}}\right|_{4}^{2}+\left|\check{Y}_{0}^{T}\right|_{4}^{2}\right\} \\
& =C_{7}(T) \exp \left\{\left|\sum_{i=1}^{m_{n}}\left(Z_{t_{i-1}}^{t_{i}}-z_{t_{i-1}}^{t_{i}}\right)-\check{Y}_{0}^{T}\right|_{4}^{2}+\left|\check{Y}_{0}^{T}\right|_{4}^{2}\right\}
\end{aligned}
$$

where $C_{7}(T)$ is a constant. The estimate (17) can then be obtained from the last inequality combined with (6).

Let us next prove:

Lemma. The limit

$$
\bar{Y}_{s}^{t}=\lim _{\delta_{n} \rightarrow 0} \sum_{k=1}^{m_{n}} x_{0}^{t_{k-1}} \check{Y}_{t_{k-1}}^{t_{k}}\left(x_{0}^{t_{k}}\right)^{-1}
$$

exists in $|\cdot|_{4}$ when $0 \leq s \leq t \leq T$ and it does not depend on the sequence of partitions $\left\{\Delta_{n}[s, t]\right\}$.

Remark. We call the limit in the formula (19) a stochastic integral and denote it $\int_{s}^{t} x_{0}^{\tau} d \check{Y}_{0}^{\tau}\left(x_{0}^{\tau}\right)^{-1}$.

Proof of the Lemma. Exactly as in [2] and [3] it is sufficient to show that the expression

$$
\left|\sum_{k=1}^{m_{n}} x_{0}^{t_{k-1}} \check{Y}_{t_{k-1}}^{t_{k}}\left(x_{0}^{t_{k}}\right)^{-1}-\sum_{k=1}^{m_{n}} \sum_{i=1}^{r_{k}} x_{0}^{s_{i-1}^{k}} \check{Y}_{s_{i-1}^{k}}^{s_{i}^{k}}\left(x_{0}^{s_{i}^{k}}\right)^{-1}\right|_{4}
$$


tends to zero when $\delta_{n} \rightarrow 0$. Here $\Delta_{n}[s, t]=\left\{s=t_{0} \leq t_{1} \leq \cdots \leq t_{m_{n}}=t\right\}$, $\Delta_{r}[s, t]=\cup_{k=1}^{m_{n}} \Delta_{r_{k}}\left[t_{k-1}, t_{k}\right], \Delta_{r_{k}}\left[t_{k-1}, t_{k}\right]=\left\{t_{k-1}=s_{0}^{k} \leq s_{1}^{k} \leq \cdots \leq s_{r_{k}}^{k}=t_{k}\right\}$. Using (9) we can write the expression (20) in another form

$$
\begin{aligned}
& \left|\sum_{k=1}^{m_{n}} \sum_{i=1}^{r_{k}} x_{0}^{t_{k-1}} \check{Y}_{s_{i-1}^{k}}^{s_{i}^{k}}\left(x_{0}^{t_{k}}\right)^{-1}-\sum_{k=1}^{m_{n}} \sum_{i=1}^{r_{k}} x_{0}^{s_{i-1}^{k}} \check{Y}_{s_{i-1}^{k}}^{s_{i}^{k}}\left(x_{0}^{s_{i}^{k}}\right)^{-1}\right|_{4}^{2} \\
& \leq 2\left|\sum_{k=1}^{m_{n}} \sum_{i=1}^{r_{k}}\left(x_{0}^{t_{k-1}}-x_{0}^{s_{i-1}^{k}}\right) \check{Y}_{s_{i-1}^{k}}^{s_{i}^{k}}\left(x_{0}^{t_{k}}\right)^{-1}\right|_{4}^{2} \\
& +2\left|\sum_{k=1}^{m_{n}} \sum_{i=1}^{r_{k}} x_{0}^{s_{i-1}^{k}} \check{Y}_{s_{i-1}^{k}}^{s_{i}^{k}}\left[\left(x_{0}^{t_{k}}\right)^{-1}-\left(x_{0}^{s_{i}^{k}}\right)^{-1}\right]\right|_{4}^{2} \\
& \leq 2 C_{1}(T) \sum_{k=1}^{m_{n}} \sum_{i=1}^{r_{k}}\left|\left(x_{0}^{t_{k-1}}-x_{0}^{s_{i-1}^{k}}\right) \check{Y}_{s_{i-1}^{k}}^{s_{i}^{k}}\right|_{4}^{2} \\
& +2 C_{2}(T) \sum_{k=1}^{m_{n}} \sum_{i=1}^{r_{k}}\left|\check{Y}_{s_{i-1}^{k}}^{s_{i}^{k}}\left[\left(x_{0}^{t_{k}}\right)^{-1}-\left(x_{0}^{s_{i}^{k}}\right)^{-1}\right]\right|_{4}^{2} \\
& \leq C_{8}(T)\left(\left|\sum_{k=1}^{m_{n}} x_{0}^{t_{k-1}} \check{Y}_{t_{k-1}}^{t_{k}}-\sum_{k=1}^{m_{n}} \sum_{i=1}^{r_{k}} x_{0}^{s_{i-1}^{k}} \check{Y}_{s_{i-1}^{k}}^{s_{i}^{k}}\right|_{4}^{2}\right. \\
& \left.+\left|\sum_{k=1}^{m_{n}} \sum_{i=1}^{r_{k}} \check{Y}_{s_{i-1}^{k}}^{s_{i}^{k}}\left(x_{0}^{s_{i}^{k}}\right)^{-1}-\sum_{k=1}^{m_{n}} \check{Y}_{t_{k-1}}^{t_{k}}\left(x_{0}^{t_{k}}\right)^{-1}\right|_{4}^{2}\right)
\end{aligned}
$$

Here $C_{8}(T)$ is a constant. The expression in the brackets on the right hand side of the inequality (21) consists of two terms. The first one is the difference between the integral sums for the left stochastic integral $(\ell) \int_{s}^{t} x_{0}^{\tau} d \check{Y}_{0}^{\tau}$ and the second is the difference between the corresponding integral sums for the right stochastic integral $(r) \int_{s}^{t} d \check{Y}_{0}^{\tau}\left(x_{0}^{\tau}\right)^{-1}$. Both these integrals exist and their definitions do not depend on the decreasing sequence of partitions $\left\{\Delta_{n}[s, t]\right\}$ (because of the property (12) and results of paper [2]). Therefore the right hand side of the inequality (21) and the expression (20) tend to zero when $\delta_{n} \rightarrow 0$.

Passing to the proof of Theorem 1 let us consider the term

$$
Z_{s}^{t}(n)=\prod_{k=1}^{m_{n}} x_{0}^{t_{k-1}}(n) Z_{t_{k-1}}^{t_{k}}\left(x_{0}^{t_{k}}(n)\right)^{-1}
$$

If we prove that the following limit

$$
\bar{X}_{s}^{t}=\lim _{\delta_{n} \rightarrow 0} Z_{s}^{t}(n)
$$

exists in $G_{2}(H, \Omega)$ and does not depend on the sequence of partitions $\left\{\Delta_{n}[s, t]\right\}$ then from the condition (5) and the property (10) it will follow the existence, in 
$G_{2}(H, \Omega)$, of the limit

$$
X_{s}^{t}=\lim _{\delta_{n} \rightarrow 0}\left(x_{0}^{s}(n)\right)^{-1} Z_{s}^{t}(n) x_{0}^{t}(n)=\lim _{\delta_{n} \rightarrow 0} \prod_{k=1}^{m_{n}} Z_{t_{k-1}}^{t_{k}}
$$

finishing the proof of the theorem as well.

To prove (23) let us note that $\check{Y}_{s}^{t}$ and $\bar{Y}_{s}^{t}$ are continuous from the left or from the right (depending on $\tau$ ) at every point $\tau \in[0, T]$ simultaneously and according to the lemma they satisfy the conditions (13)-(15). By [4] the following limit exists in $G_{2}(H, \Omega)$ and does not depend on the sequence of partitions $\left\{\Delta_{n}[s, t]\right\}$ :

$$
\lim _{\delta_{n} \rightarrow 0} \prod_{k=1}^{m_{n}}\left(\bar{Y}_{t_{k-1}}^{t_{k}}+I\right)
$$

Let us show that

$$
\lim _{\delta_{n} \rightarrow 0} \prod_{k=1}^{m_{n}}\left(\bar{Y}_{t_{k-1}}^{t_{k}}+I\right)=\lim _{\delta_{n} \rightarrow 0} Z_{s}^{t}(n)=\bar{X}_{s}^{t} .
$$

To prove this equality, as in [4], it is sufficient to consider the decompositions $\Delta_{n}[s, t]$ from (20) and use the following estimate

$$
\begin{aligned}
& \left|\prod_{k=1}^{m_{n}}\left(\bar{Y}_{t_{k-1}}^{t_{k}}+I\right)-\prod_{k=1}^{m_{n}} x_{0}^{t_{k-1}}(n) Z_{t_{k-1}}^{t_{k}}\left(x_{0}^{t_{k}}(n)\right)^{-1}\right|_{4}^{2} \\
& =\mid \sum_{k=1}^{m_{n}} \prod_{i=1}^{k-1}\left(\bar{Y}_{t_{i-1}}^{t_{i}}+I\right)\left[\bar{Y}_{t_{k-1}}^{t_{k}}-x_{0}^{t_{k-1}}(n)\left(Z_{t_{k-1}}^{t_{k}}-z_{t_{k-1}}^{t_{k}}\right)\left(x_{0}^{t_{k}}(n)\right)^{-1}\right] \\
& \left.\cdot \prod_{i=k+1}^{m_{n}} x_{0}^{t_{i-1}}(n) Z_{t_{i-1}}^{t_{i}}\left(x_{0}^{t_{i}}(n)\right)^{-1}\right|_{4} ^{2} \\
& \leq 3 \mid \sum_{k=1}^{m_{n}} \prod_{i=1}^{k-1}\left(\bar{Y}_{t_{i-1}}^{t_{i}}+I\right) x_{0}^{t_{k-1}}(n)\left[\check{Y}_{t_{k-1}}^{t_{k}}-Z_{t_{k-1}}^{t_{k}}+z_{t_{k-1}}^{t_{k}}\right] \\
& \left.\cdot\left(x_{0}^{t_{k}}(n)\right)^{-1} \prod_{i=k+1}^{m_{n}} x_{0}^{t_{i-1}}(n) Z_{t_{i-1}}^{t_{i}}\left(x_{0}^{t_{i}}(n)\right)^{-1}\right|_{4} ^{2} \\
& +3 \mid \sum_{k=1}^{m_{n}} \prod_{i=1}^{k-1}\left(\bar{Y}_{t_{i-1}}^{t_{i}}+I\right) \int_{t_{k-1}}^{t_{k}}\left(x_{0}^{\tau}-x_{0}^{t_{k-1}}(n)\right) d \check{Y}_{0}^{\tau}\left(x_{0}^{t_{k}}(n)\right)^{-1} \\
& \left.\cdot \prod_{i=k+1}^{m_{n}} x_{0}^{t_{i-1}}(n) Z_{t_{i-1}}^{t_{i}}\left(x_{0}^{t_{i}}(n)\right)^{-1}\right|_{4} ^{2}+3 \mid \sum_{k=1}^{m_{n}} \prod_{i=1}^{k-1}\left(\bar{Y}_{t_{i-1}}^{t_{i}}+I\right) \int_{t_{k-1}}^{t_{k}} x_{0}^{\tau} d \check{Y}_{0}^{\tau} \\
& \left.\cdot\left(\left(x_{0}^{\tau}\right)^{-1}-\left(x_{0}^{t_{k}}(n)\right)^{-1}\right) \prod_{i=k+1}^{m_{n}} x_{0}^{t_{i-1}}(n) Z_{t_{i-1}}^{t_{i}}\left(x_{0}^{t_{i}}(n)\right)^{-1}\right|_{4} ^{2} \cdot
\end{aligned}
$$


Since $\bar{Y}_{s}^{t}$ admits the properties (13)-(15) we can rewrite the first term on the right hand side of (26) in the form

$$
\begin{aligned}
& 3 \sum_{k=1}^{m_{n}} \mid \prod_{i=1}^{k-1}\left(\bar{Y}_{t_{i-1}}^{t_{i}}+I\right) x_{0}^{t_{k-1}}(n)\left[\check{Y}_{t_{k-1}}^{t_{k}}-Z_{t_{k-1}}^{t_{k}}+z_{t_{k-1}}^{t_{k}}\right]\left(x_{0}^{t_{k}}(n)\right)^{-1} \\
& \left.\cdot \prod_{i=k+1}^{m_{n}} x_{0}^{t_{i-1}}(n) Z_{t_{i-1}}^{t_{i}}\left(x_{0}^{t_{i}}(n)\right)^{-1}\right|_{4} ^{2}+\sum_{k \neq j} \mathbf{E} \operatorname{trace}\left\{\prod_{i=1}^{k-1}\left(\bar{Y}_{t_{i-1}}^{t_{i}}+I\right) x_{0}^{t_{k-1}}(n)\right. \\
& \cdot\left[\check{Y}_{t_{k-1}}^{t_{k}}-Z_{t_{k-1}}^{t_{k}}+z_{t_{k-1}}^{t_{k}}\right]\left(x_{0}^{t_{k}}(n)\right)^{-1} \prod_{i=k+1}^{j-1} x_{0}^{t_{i-1}}(n) Z_{t_{i-1}}^{t_{i}}\left(x_{0}^{t_{i}}(n)\right)^{-1} x_{0}^{t_{j-1}}(n) \\
& \left.\cdot\left(Z_{t_{j-1}}^{t_{j}}-z_{t_{j-1}}^{t_{j}}\right)\left(x_{0}^{t_{j}}(n)\right)^{-1} \prod_{i=j+1}^{m_{n}} x_{0}^{t_{i-1}}(n) Z_{t_{i-1}}^{t_{i}}\left(x_{0}^{t_{i}}(n)\right)^{-1}\right\}^{*} \\
& \cdot\left\{\prod_{i=1}^{k-1}\left(\bar{Y}_{t_{i-1}}^{t_{i}}+I\right) \bar{Y}_{t_{k-1}}^{t_{k}} \prod_{i=k+1}^{j-1}\left(\bar{Y}_{t_{i-1}}^{t_{i}}+I\right) x_{0}^{t_{j-1}}(n)\left[\check{Y}_{t_{j-1}}^{t_{j}}-Z_{t_{j-1}}^{t_{j}}+z_{t_{j-1}}^{t_{j}}\right]\right. \\
& \left.\cdot\left(x_{0}^{t_{j}}(n)\right)^{-1} \prod_{i=j+1}^{m_{n}} x_{0}^{t_{i-1}}(n) Z_{t_{i-1}}^{t_{i}}\left(x_{0}^{t_{i}}(n)\right)^{-1}\right\} .
\end{aligned}
$$

The first term in (27) is, by estimates (8), (16), (17) and the properties of the norms $|\cdot|_{4}$ and $|\cdot|_{5}$, bounded by the estimate

$$
\begin{aligned}
& 3 C_{5}(T) C_{3}^{2}(T) C_{4}(T) C_{6}(T) \sum_{k=1}^{m_{n}}\left|\check{Y}_{t_{k-1}}^{t_{k}}-Z_{t_{k-1}}^{t_{k}}+z_{t_{k-1}}^{t_{k}}\right|_{4}^{2} \\
& =C_{9}(T)\left|\sum_{k=1}^{m_{n}}\left(Z_{t_{k-1}}^{t_{k}}-z_{t_{k-1}}^{t_{k}}\right)-\check{Y}_{s}^{t}\right|_{4}^{2}
\end{aligned}
$$

tending to zero as $n \rightarrow \infty$ due to the condition (6).

In order to estimate the second term in (27) we note that from the CauchySchwarz inequality, the estimates $(9),(16),(17)$ and the evident inequality $\sqrt{a^{2}+b^{2}}$ $\leq a+b$ when $a \geq 0, b \geq 0$ it follows that its modulus is bounded by the estimates

$$
\begin{aligned}
& \sum_{k \neq j} \mathbf{E}\left\{\mid \prod_{i=1}^{k-1}\left(\bar{Y}_{t_{i-1}}^{t_{i}}+I\right) x_{0}^{t_{k-1}}(n)\left[\check{Y}_{t_{k-1}}^{t_{k}}-Z_{t_{k-1}}^{t_{k}}+z_{t_{k-1}}^{t_{k}}\right]\left(x_{0}^{t_{k}}(n)\right)^{-1}\right. \\
& \cdot \prod_{i=k+1}^{j-1} x_{0}^{t_{i-1}}(n) Z_{t_{i-1}}^{t_{i}}\left(x_{0}^{t_{i}}(n)\right)^{-1} x_{0}^{t_{j-1}}(n)\left(Z_{t_{j-1}}^{t_{j}}-z_{t_{j-1}}^{t_{j}}\right)\left(x_{0}^{t_{j}}(n)\right)^{-1} \\
& \left.\cdot \prod_{i=j+1}^{m_{n}} x_{0}^{t_{i-1}}(n) Z_{t_{i-1}}^{t_{i}}\left(x_{0}^{t_{i}}(n)\right)^{-1}\right|_{3} \mid \prod_{i=1}^{k-1}\left(\bar{Y}_{t_{i-1}}^{t_{i}}+I\right) \bar{Y}_{t_{k-1}}^{t_{k}}
\end{aligned}
$$




$$
\begin{aligned}
& \cdot \prod_{i=k+1}^{j-1}\left(\bar{Y}_{t_{i-1}}^{t_{i}}+I\right) x_{0}^{t_{j-1}}(n)\left[\check{Y}_{t_{j-1}}^{t_{j}}-Z_{t_{j-1}}^{t_{j}}+z_{t_{j-1}}^{t_{j}}\right] \\
& \left.\left.\cdot\left(x_{0}^{t_{j}}(n)\right)^{-1} \prod_{i=j+1}^{m_{n}} x_{0}^{t_{i-1}}(n) Z_{t_{i-1}}^{t_{i}}\left(x_{0}^{t_{i}}(n)\right)^{-1}\right|_{3}\right\} \leq\left(\sum_{k \neq j} \mid \prod_{i=1}^{k-1}\left(\bar{Y}_{t_{i-1}}^{t_{i}}+I\right)\right. \\
& \cdot x_{0}^{t_{k-1}}(n)\left[\check{Y}_{t_{k-1}}^{t_{k}}-Z_{t_{k-1}}^{t_{k}}+z_{t_{k-1}}^{t_{k}}\right]\left(x_{0}^{t_{k}}(n)\right)^{-1} \\
& \cdot \prod_{i=k+1}^{j-1} x_{0}^{t_{i-1}}(n) Z_{t_{i-1}}^{t_{i}}\left(x_{0}^{t_{i}}(n)\right)^{-1} x_{0}^{t_{j-1}}(n)\left(Z_{t_{j-1}}^{t_{j}}-z_{t_{j-1}}^{t_{j}}\right)\left(x_{0}^{t_{j}}(n)\right)^{-1} \\
& \left.\left.\cdot \prod_{i=j+1}^{m_{n}} x_{0}^{t_{i-1}}(n) Z_{t_{i-1}}^{t_{i}}\left(x_{0}^{t_{i}}(n)\right)^{-1}\right|_{4} ^{2}\right)^{1 / 2} \\
& \cdot\left(\sum_{k \neq j} \mid \prod_{i=1}^{k-1}\left(\bar{Y}_{t_{i-1}}^{t_{i}}+I\right) \bar{Y}_{t_{k-1}}^{t_{k}} \prod_{i=k+1}^{j-1}\left(\bar{Y}_{t_{i-1}}^{t_{i}}+I\right) x_{0}^{t_{j-1}}(n)\left[\check{Y}_{t_{j-1}}^{t_{j}}-Z_{t_{j-1}}^{t_{j}}+z_{t_{j-1}}^{t_{j}}\right]\right. \\
& \left.\left.\cdot\left(x_{0}^{t_{j}}(n)\right)^{-1} \prod_{i=j+1}^{m_{n}} x_{0}^{t_{i-1}}(n) Z_{t_{i-1}}^{t_{i}}\left(x_{0}^{t_{i}}(n)\right)^{-1}\right|_{4} ^{2}\right)^{1 / 2} \\
& \leq C_{5}(T) C_{3}^{2}(T) C_{4}^{3}(T) C_{6}(T)\left(\sum_{k=1}^{m_{n}} \sum_{j=1}^{k-1}\left|\check{Y}_{t_{k-1}}^{t_{k}}-Z_{t_{k-1}}^{t_{k}}+z_{t_{k-1}}^{t_{k}}\right|_{4}^{2}\left|Z_{t_{j-1}}^{t_{j}}-z_{t_{j-1}}^{t_{j}}\right|_{4}^{2}\right)^{1 / 2} \\
& \cdot\left(\sum_{k=1}^{m_{n}} \sum_{j=1}^{k-1}\left|\bar{Y}_{t_{k-1}}^{t_{k}}\right|_{4}^{2}\left|\check{Y}_{t_{j-1}}^{t_{j}}-Z_{t_{j-1}}^{t_{j}}+z_{t_{j-1}}^{t_{j}}\right|_{4}^{2}\right)^{1 / 2} \leq C_{10}(T)\left|\check{Y}_{s}^{t}-\sum_{k=1}^{m_{n}}\left(Z_{t_{k-1}}^{t_{k}}-z_{t_{k-1}}^{t_{k}}\right)\right|_{4}^{2} \\
& \cdot\left(\left|\check{Y}_{s}^{t}-\sum_{k=1}^{m_{n}}\left(Z_{t_{k-1}}^{t_{k}}-z_{t_{k-1}}^{t_{k}}\right)\right|_{4}+\left|\check{Y}_{s}^{t}\right|_{4}\right)\left|\check{Y}_{s}^{t}\right|_{4}
\end{aligned}
$$

tending to zero as $n \rightarrow \infty$ due to the condition (6). Here $C_{10}(T)$ is a constant. Thus the first term on the right hand side of (26) tends to zero when $n \rightarrow \infty$. In order to estimate the second term let us rewrite it in the form

$$
\begin{aligned}
& \sum_{k=1}^{m_{n}} \mid \prod_{i=1}^{k-1}\left(\bar{Y}_{t_{i-1}}^{t_{i}}+I\right)\left[\int_{t_{k-1}}^{t_{k}}\left(x_{0}^{\tau}-x_{0}^{t_{k-1}}(n)\right) d \check{Y}_{0}^{\tau}\left(x_{0}^{t_{k}}(n)\right)^{-1}\right] \\
& \left.\cdot \prod_{i=k+1}^{m_{n}} x_{0}^{t_{i-1}}(n) Z_{t_{i-1}}^{t_{i}}\left(x_{0}^{t_{i}}(n)\right)^{-1}\right|_{4} ^{2}+\sum_{k \neq j} \mathbf{E} \operatorname{trace}\left\{\prod_{i=1}^{k-1}\left(\bar{Y}_{t_{i-1}}^{t_{i}}+I\right)\right. \\
& \cdot\left[\int_{t_{k-1}}^{t_{k}}\left(x_{0}^{\tau}-x_{0}^{t_{k-1}}(n)\right) d \check{Y}_{0}^{\tau}\left(x_{0}^{t_{k}}(n)\right)^{-1}\right] \prod_{i=k+1}^{j-1} x_{0}^{t_{i-1}}(n) Z_{l_{i-1}}^{t_{i}}\left(x_{0}^{t_{i}}(n)\right)^{-1}
\end{aligned}
$$




$$
\begin{aligned}
& \left.\cdot x_{0}^{t_{i-1}}(n)\left(Z_{t_{i-1}}^{t_{i}}-z_{t_{i-1}}^{t_{i}}\right)\left(x_{0}^{t_{j}}(n)\right)^{-1} \prod_{i=j+1}^{m_{n}} x_{0}^{t_{i-1}}(n) Z_{t_{i-1}}^{t_{i}}\left(x_{0}^{t_{i}}(n)\right)^{-1}\right\}^{*} \\
& \cdot\left\{\prod_{i=1}^{k-1}\left(\bar{Y}_{t_{i-1}}^{t_{i}}+I\right) \bar{Y}_{t_{k-1}}^{t_{k}} \prod_{i=k+1}^{j-1}\left(\bar{Y}_{t_{i-1}}^{t_{i}}+I\right)\right. \\
& \left.\cdot\left[\int_{t_{j-1}}^{t_{j}}\left(x_{0}^{\tau}-x_{0}^{t_{j-1}}(n)\right) d \check{Y}_{0}^{\tau}\left(x_{0}^{t_{j}}(n)\right)^{-1}\right] \prod_{i=j+1}^{m_{n}} x_{0}^{t_{i-1}}(n) Z_{t_{i-1}}^{t_{i}}\left(x_{0}^{t_{i}}(n)\right)^{-1}\right\} .
\end{aligned}
$$

Using the estimates which were proved as indicated above we can see that the first term in (29) is bounded by

$$
\begin{aligned}
& C_{11}(T)\left(\sum_{k=1}^{m_{n}}\left|\int_{t_{k-1}}^{t_{k}}\left(x_{0}^{\tau}-x_{0}^{t_{k-1}}\right) d \check{Y}_{0}^{\tau}\right|_{4}^{2}+\sum_{k=1}^{m_{n}}\left|\int_{t_{k-1}}^{t_{k}}\left(x_{0}^{t_{k-1}}-x_{0}^{t_{k-1}}(n)\right) d \check{Y}_{0}^{\tau}\right|_{4}^{2}\right) \\
& \leq C_{11}(T)\left(\left|(\ell) \int_{s}^{t} x_{0}^{\tau} d \check{Y}_{0}^{\tau}-\sum_{k=1}^{m_{n}} x_{0}^{t_{k-1}} \check{Y}_{t_{k-1}}^{t_{k}}\right|_{4}^{2}\right)+\sup _{k}\left|x_{0}^{t_{k-1}}-x_{0}^{t_{k-1}}(n)\right|_{2}\left|\check{Y}_{s}^{t}\right|_{4}^{2}
\end{aligned}
$$

tending to zero as $n \rightarrow \infty$ due to the condition (5) and property (12), which is sufficient for the existence of the left stochastic integral $(\ell) \int_{s}^{t} x_{0}^{\tau} d \check{Y}_{0}^{\tau}$ and its independence of the sequence of partitions $\left\{\Delta_{n}[s, t]\right\}$. Now it is easy to see that the integral $(\ell) \int_{s}^{t} x_{0}^{\tau} d \check{Y}_{0}^{\tau}$ is additive as a function of the intervals $\left[t_{k-1}, t_{k}\right]$.

Analogously, the modulus of the second term in (29) is bounded by

$$
\begin{aligned}
& \sum_{k \neq j} \mathbf{E} \mid \prod_{i=1}^{k-1}\left(\bar{Y}_{t_{i-1}}^{t_{i}}+I\right)\left[\int_{t_{k-1}}^{t_{k}}\left(x_{0}^{\tau}-x_{0}^{t_{k-1}}(n)\right) d \check{Y}_{0}^{\tau}\left(x_{0}^{t_{k}}(n)\right)^{-1}\right] \\
& \cdot \prod_{i=k+1}^{m_{n}} x_{0}^{t_{i-1}}(n) Z_{t_{i-1}}^{t_{i}}\left(x_{0}^{t_{i}}(n)\right)^{-1} x_{0}^{t_{j-1}}(n)\left(Z_{t_{j-1}}^{t_{j}}-z_{t_{j-1}}^{t_{j}}\right)\left(x_{0}^{t_{j}}(n)\right)^{-1} \\
& \cdot \prod_{i=j+1}^{m_{n}} x_{0}^{t_{i-1}}(n) Z_{t_{i-1}}^{t_{i}}\left(x_{0}^{t_{i}}(n)\right)^{-1}\left|{ }_{3}\right| \prod_{i=1}^{k-1}\left(\bar{Y}_{t_{i-1}}^{t_{i}}+I\right) \bar{Y}_{t_{k-1}}^{t_{k}} \prod_{i=k+1}^{j-1}\left(\bar{Y}_{t_{i-1}}^{t_{i}}+I\right) \\
& \left.\cdot\left[\int_{t_{j-1}}^{t_{j}}\left(x_{0}^{\tau}-x_{0}^{t_{j-1}}(n)\right) d \check{Y}_{0}^{\tau}\left(x_{0}^{t_{j}}(n)\right)^{-1}\right] \prod_{i=j+1}^{m_{n}} x_{0}^{t_{i-1}}(n) Z_{t_{i-1}}^{t_{i}}\left(x_{0}^{t_{i}}(n)\right)^{-1}\right|_{3} \\
& \leq C_{12}(T)\left\{\sum _ { k \neq j } \left(\left|\int_{t_{k-1}}^{t_{k}}\left(x_{0}^{\tau}-x_{0}^{t_{k-1}}\right) d \check{Y}_{0}^{\tau}\right|_{4}^{2}\right.\right. \\
& \left.\left.+\left|\int_{t_{k-1}}^{t_{k}}\left(x_{0}^{t_{k-1}}-x_{0}^{t_{k-1}}(n)\right) d \check{Y}_{0}^{\tau}\right|_{4}^{2}\right)\left|Z_{t_{j-1}}^{t_{j}}-z_{t_{j-1}}^{t_{j}}\right|_{4}^{2}\right\}^{1 / 2}
\end{aligned}
$$




$$
\begin{aligned}
& \cdot\left\{\sum_{k \neq j}\left|\check{Y}_{t_{k-1}}^{t_{k}}\right|_{4}^{2}\left(\left|\int_{t_{j-1}}^{t_{j}}\left(x_{0}^{\tau}-x_{0}^{t_{j-1}}\right) d \check{Y}_{0}^{\tau}\right|_{4}^{2}+\left|\int_{t_{j-1}}^{t_{j}}\left(x_{0}^{t_{j-1}}-x_{0}^{t_{j-1}}(n)\right) d \check{Y}_{0}^{\tau}\right|_{4}^{2}\right)\right\}^{1 / 2} \\
& \leq C_{12}(T)\left(\left|(\ell) \int_{s}^{t} x_{0}^{\tau} d \check{Y}_{0}^{\tau}-\sum_{k=1}^{m_{n}} x_{0}^{t_{k-1}} \check{Y}_{t_{k-1}}^{t_{k}}\right|_{4}^{2}+\sup _{k}\left|x_{0}^{t_{k-1}}-x_{0}^{t_{k-1}}(n)\right|_{2}^{2}\left|\check{Y}_{s}^{t}\right|_{4}^{2}\right)^{1 / 2} \\
& \cdot\left(\left|\sum_{j=1}^{m_{n}}\left(Z_{t_{j-1}}^{t_{j}}-z_{t_{j-1}}^{t_{j}}\right)-\check{Y}_{s}^{t}\right|_{4}+\left|\check{Y}_{s}^{t}\right|_{4}\right) \\
& \cdot\left(\left|(\ell) \int_{s}^{t} x_{0}^{\tau} d \check{Y}_{0}^{\tau}-\sum_{j=1}^{m_{n}} x_{0}^{t_{j-1}} \check{Y}_{t_{j-1}}^{t_{j}}\right|_{4}^{2}+\sup _{j}\left|x_{0}^{t_{j-1}}-x_{0}^{t_{j-1}}(n)\right|_{2}^{2}\left|\check{Y}_{s}^{t}\right|_{4}^{2}\right)^{1 / 2}\left|\check{Y}_{s}^{t}\right|_{4}
\end{aligned}
$$

where $C_{12}(T)$ is a constant. Analogously, the right hand side of the last inequality tends to zero when $n \rightarrow \infty$.

Thus the second term on the right hand side of (26) tends to zero when $n \rightarrow \infty$, too.

The third term on the right hand side of (26) can be estimated in the same way as as the second term if one uses the property (10) instead of the condition (5) when considering the right stochastic integral $(r) \int_{s}^{t} d \check{Y}_{0}^{\tau}\left(x_{0}^{\tau}\right)^{-1}$; its necessary properties are guaranteed by the relation (12) and the results of the paper [2].

Remark 1. If we omit in the condition (5) the property that $x_{s}^{t} \in G_{2}(H)$ we must consider in (1) the convergence in mean square with respect to the norm $|\cdot|_{2}$ instead of $|\cdot|_{3}$. The convergence in (23) remains valid with respect to the norm $|\cdot|_{4}$.

Remark 2. If $Z_{s}^{t}$ and $x_{s}^{t}$ are uniformly continuous, i.e., $\left|Z_{s}^{t}-I\right|_{4} \rightarrow 0$, $\left|x_{s}^{t}-I\right|_{2} \rightarrow 0$ when $t-s \rightarrow 0$, then Theorem 1 is true without the condition (5). In fact, the properties (8) in that case are evident and the properties (9) are replaced by the next relation when $\delta_{n} \rightarrow 0$ :

$$
\sup _{t_{k-1} \leq \tau \leq t_{k}}\left\{\left|x_{0}^{\tau}-x_{0}^{t_{k-1}}(n)\right|_{2},\left|\left(x_{0}^{\tau}\right)^{-1}-\left(x_{0}^{t_{k-1}}(n)\right)^{-1}\right|_{2}\right\} \longrightarrow 0
$$

which can be proved by rule of contradiction. Then the property (30) is used in estimating the expression (29) instead of the condition (5) and the property (10).

Theorem 2. If the system $Z_{s}^{t}, 0 \leq s \leq t \leq T \leq \infty$ satisfies the conditions (4)-(6), then the formulas

and

$$
X_{s}^{t}=\lim _{\delta_{n} \rightarrow 0} \prod_{k=1}^{m_{n}} Z_{t_{k-1}}^{t_{k}}=\lim _{\delta_{n} \rightarrow 0} \prod_{k=1}^{m_{n}}\left(\check{Y}_{t_{k-1}}^{t_{k}}+z_{t_{k-1}}^{t_{k}}\right)
$$




$$
\check{Y}_{s}^{t}=\lim _{\delta_{n} \rightarrow 0} \sum_{k=1}^{m_{n}}\left(X_{t_{k-1}}^{t_{k}}-x_{t_{k-1}}^{t_{k}}\right)
$$

where $x_{s}^{t}=\mathbf{E} X_{s}^{t}$, are true. Here convergence is with respect to the norm $|\cdot|_{4}$ and these limits do not depend on the decreasing sequence of partitions $\left\{\Delta_{n}[s, t]\right\}$.

Proof. First of all let us note that due to the inequalities (9) and (17) the following inequality is true for every partition $\Delta_{n}[s, t]$ :

$$
\alpha=\sup _{u, p, r}\left|\prod_{i=p}^{r} Z_{t_{i-1}}^{t_{i}}\right|_{5}<\infty, \quad 1 \leq p \leq r \leq m_{n} .
$$

Let us consider the term

$$
\begin{aligned}
& \prod_{i=p}^{r}\left(x_{0}^{t_{i-1}}(n) \check{Y}_{t_{i-1}}^{t_{i}}\left(x_{0}^{t_{i}}(n)\right)^{-1}+I\right) \\
& =\prod_{i=p}^{r} x_{0}^{t_{i-1}}(n)\left(\check{Y}_{t_{i-1}}^{t_{i}}+z_{t_{i-1}}^{t_{i}}\right)\left(x_{0}^{t_{i}}(n)\right)^{-1} \\
& =x_{0}^{t_{p-1}}(n)\left(\prod_{i=p}^{r}\left(\check{Y}_{t_{i-1}}^{t_{i}}+z_{t_{i-1}}^{t_{i}}\right)\left(x_{0}^{t_{r}}(n)\right)^{-1}\right)
\end{aligned}
$$

and note that, due to the estimates (9), it is enough to prove that the term (34) is bounded in order to show that

$$
\beta=\sup _{u, p, r}\left|\prod_{i=p}^{r}\left(\check{Y}_{t_{i-1}}^{t_{i}}+z_{t_{i-1}}^{t_{i}}\right)\right|_{5}<\infty
$$

holds. Therefore, using the known properties of the norms $|\cdot|_{4}$ and $|\cdot|_{5}(\mathrm{cf} .[2])$ and the properties (9), (13) and (14) we obtain the next estimate

$$
\begin{aligned}
& \left|\prod_{i=p}^{r} x_{0}^{t_{i-1}}(n) \check{Y}_{t_{i-1}}^{t_{i}}\left(x_{0}^{t_{i}}(n)\right)^{-1}+I\right|_{5}^{2} \leq \prod_{i=p}^{r}\left(\left|x_{0}^{t_{i}}(n)\right|_{5}^{2}\left|\check{Y}_{t_{i-1}}^{t_{i}}\right|_{4}^{2}\left|\left(x_{0}^{t_{i}}(n)\right)^{-1}\right|_{5}^{2}+1\right) \\
& \leq \exp \left\{C_{1}(T) \sum_{i=p}^{r}\left|\check{Y}_{t_{i-1}}^{t_{i}}\right|_{4}^{2}\right\} \leq \exp \left\{C_{1}(T)\left|\check{Y}_{0}^{T}\right|_{4}^{2}\right\}<\infty
\end{aligned}
$$

for the expression (34). By using once more the estimate (9) we then easily obtain the inequality (35). Here $C_{1}(T)$ is some constant. 
Let us now estimate the difference

$$
\begin{aligned}
& \left|\prod_{k=1}^{m_{n}} Z_{t_{k-1}}^{t_{k}}-\prod_{k=1}^{m_{n}}\left(\check{Y}_{t_{k-1}}^{t_{k}}+z_{t_{k-1}}^{t_{k}}\right)\right|_{4}^{2} \\
& =\left|\sum_{k=1}^{m_{n}} \prod_{i=1}^{k-1} Z_{t_{i-1}}^{t_{i}}\left(Z_{t_{k-1}}^{t_{k}}-z_{t_{k-1}}^{t_{k}}-\check{Y}_{t_{k-1}}^{t_{k}}\right) \prod_{i=k+1}^{m_{n}}\left(\check{Y}_{t_{i-1}}^{t_{i}}+z_{t_{i-1}}^{t_{i}}\right)\right|_{4}^{2} \\
& =\sum_{k=1}^{m_{n}}\left|\prod_{i=1}^{k-1} Z_{t_{i-1}}^{t_{i}}\left(Z_{t_{k-1}}^{t_{k}}-z_{t_{k-1}}^{t_{k}}-\check{Y}_{t_{k-1}}^{t_{k}}\right) \prod_{i=k+1}^{m_{n}}\left(\check{Y}_{t_{i-1}}^{t_{i}}+z_{t_{i-1}}^{t_{i}}\right)\right|_{4}^{2} \\
& +\sum_{k \neq j} \operatorname{trace} \mathbf{E}\left(\prod_{i=1}^{k-1} Z_{t_{i-1}}^{t_{i}}\left(Z_{t_{k-1}}^{t_{k}}-z_{t_{k-1}}^{t_{k}}-\check{Y}_{t_{k-1}}^{t_{k}}\right) \prod_{i=k+1}^{j-1}\left(\check{Y}_{t_{i-1}}^{t_{i}}+z_{t_{i-1}}^{t_{i}}\right)\right. \\
& \left.\cdot \check{Y}_{t_{j-1}}^{t_{j}} \prod_{i=j+1}^{m_{n}}\left(\check{Y}_{t_{i-1}}^{t_{i}}+z_{t_{i-1}}^{t_{i}}\right)\right) \prod_{i=1}^{k-1} Z_{t_{i-1}}^{t_{i}}\left(Z_{t_{k-1}}^{t_{k}}-z_{t_{k-1}}^{t_{k}}\right) \prod_{i=k+1}^{j-1} Z_{t_{i-1}}^{t_{i}} \\
& \cdot\left(Z_{t_{i-1}}^{t_{i}}-z_{t_{i-1}}^{t_{i}}-\check{Y}_{t_{i-1}}^{t_{i}}\right) \prod_{i=j+1}^{m_{n}}\left(\check{Y}_{t_{i-1}}^{t_{i}}+z_{t_{i-1}}^{t_{i}}\right) .
\end{aligned}
$$

By using the estimates (33), (35) and the properties (2.8), (2.10) of the norms $|\cdot|_{4}$ and $|\cdot|_{5}$ in the paper [2] we can note that the first term on the right hand side of the expression (36) is bounded by the term

$$
\begin{aligned}
& \alpha^{2} \beta^{2} \sum_{k=1}^{m_{n}}\left|Z_{t_{k-1}}^{t_{k}}-z_{t_{k-1}}^{t_{k}}-\check{Y}_{t_{k-1}}^{t_{k}}\right|_{4}^{2}=\alpha^{2} \beta^{2}\left|\sum_{k=1}^{m_{n}}\left(Z_{t_{k-1}}^{t_{k}}-z_{t_{k-1}}^{t_{k}}\right)-\check{Y}_{s}^{t}\right|_{4}^{2} \\
& =\alpha^{2} \beta^{2} \varepsilon_{n}^{2}, \text { with } \varepsilon_{n}=\left|\sum_{k=1}^{m_{n}}\left(Z_{t_{k-1}}^{t_{k}}-z_{t_{k-1}}^{t_{k}}\right)-\check{Y}_{s}^{t}\right|_{4}
\end{aligned}
$$

On the other hand, using the Cauchy-Schwarz inequality and the properties (6), (13), (14) we note that the modulus of the second term on the right hand side of the expression (36) is bounded by the term

$$
\begin{aligned}
& \left(\sum_{k \neq j} \mid \prod_{i=1}^{k-1} Z_{t_{i-1}}^{t_{i}}\left(Z_{t_{k-1}}^{t_{k}}-z_{t_{k-1}}^{t_{k}}-\check{Y}_{t_{k-1}}^{t_{k}}\right) \prod_{i=k+1}^{j-1}\left(\check{Y}_{t_{i-1}}^{t_{i}}+z_{t_{i-1}}^{t_{i}}\right) \check{Y}_{t_{i-1}}^{t_{i}}\right. \\
& \left.\left.\cdot \prod_{i=j+1}^{m_{n}}\left(\check{Y}_{t_{i-1}}^{t_{i}}+z_{t_{i-1}}^{t_{i}}\right)\right|_{4} ^{2}\right)^{1 / 2}\left(\sum_{k \neq j} \mid \prod_{i=1}^{k-1} Z_{t_{i-1}}^{t_{i}}\left(Z_{t_{k-1}}^{t_{k}}-z_{t_{k-1}}^{t_{k}}\right)\right. \\
& \left.\left.\cdot \prod_{i=k+1}^{j-1} Z_{t_{i-1}}^{t_{i}}\left(Z_{t_{j-1}}^{t_{j}}-z_{t_{j-1}}^{t_{j}}-\check{Y}_{t_{j-1}}^{t_{j}}\right) \prod_{i=j+1}^{m_{n}}\left(\check{Y}_{t_{i-1}}^{t_{i}}+z_{t_{i-1}}^{t_{i}}\right)\right|_{4} ^{2}\right)^{1 / 2}
\end{aligned}
$$




$$
\begin{aligned}
& \leq \alpha \beta^{2}\left(\sum_{k=1}^{m_{n}}\left|Z_{t_{k-1}}^{t_{k}}-z_{t_{k-1}}^{t_{k}}-\check{Y}_{t_{k-1}}^{t_{k}}\right|_{4}^{2}\right)^{1 / 2}\left(\sum_{j=1}^{m_{n}}\left|\check{Y}_{t_{j-1}}^{t_{j}}\right|_{4}^{2}\right)^{1 / 2} \\
& \cdot \alpha^{2} \beta\left(\sum_{k=1}^{m_{n}}\left|Z_{t_{k-1}}^{t_{k}}-z_{t_{k-1}}^{t_{k}}\right|_{4}^{2}\right)^{1 / 2}\left(\sum_{j=1}^{m_{n}}\left|Z_{t_{j-1}}^{t_{j}}-z_{t_{j-1}}^{t_{j}}-\check{Y}_{t_{j-1}}^{t_{j}}\right|_{4}^{2}\right)^{1 / 2} \\
& =\alpha^{3} \beta^{3} \varepsilon_{n}^{2}\left|\check{Y}_{s}^{t}\right|_{4}^{2}\left|\sum_{k=1}^{m_{n}}\left(Z_{t_{k-1}}^{t_{k}}-z_{t_{k-1}}^{t_{k}}\right)\right|_{4}^{2} \leq C_{2}(T) \varepsilon_{n}^{2} .
\end{aligned}
$$

Therefore the difference (36) is bounded by the term $\varepsilon_{n}^{2} C_{3}(T)$, which due to the condition (6) converges to zero as $n \rightarrow \infty$. Here $C_{2}(T)$ and $C_{3}(T)$ are some constants. Thus the formula (31) is proved, because, due to Theorem 1 , the first limit in this formula does not depend on the sequence of partitions $\left\{\Delta_{n}[s, t]\right\}$. In order to prove (32) let us show, first of all, that the first equality on the left hand side of

$$
\check{Y}_{s}^{t}=\lim _{\delta_{n} \rightarrow 0} \sum_{k=1}^{m_{n}}\left(x_{0}^{t_{k-1}}\right)^{-1} \bar{Y}_{t_{k-1}}^{t_{k}} x_{0}^{t_{k}}=\int_{s}^{t}\left(x_{0}^{\tau}\right)^{-1} d \bar{Y}_{0}^{\tau} x_{0}^{\tau}
$$

is true. Here the convergence is considered in the norm $|\cdot|_{4}$ and does not depend on the sequence of partitions $\left\{\Delta_{n}[s, t]\right\}$. Let us call this limit a stochastic integral and, analogously to (19), denote it accordingly. It is easy to see that the existence of the limit in (38) and its independence of the sequence of partitions $\left\{\Delta_{n}[s, t]\right\}$ may be proved in the same way as in (19). Therefore, we need to prove only the first equality in (38).

For this purpose, by using the relations (8), (14) and (19) we obtain

$$
\begin{aligned}
& \left|\check{Y}_{s}^{t}-\sum_{k=1}^{m_{n}}\left(x_{0}^{t_{k-1}}\right)^{-1} \bar{Y}_{t_{k-1}}^{t_{k}}\left(x_{0}^{t_{k}}\right)\right|_{4}^{2} \\
& =\left|\sum_{k=1}^{m_{n}} \check{Y}_{t_{k-1}}^{t_{k}}-\sum_{k=1}^{m_{n}}\left(x_{0}^{t_{k}}\right)^{-1} \int_{t_{k-1}}^{t_{k}} x_{0}^{\tau} d \check{Y}_{0}^{\tau}\left(x_{0}^{\tau}\right)^{-1} x_{0}^{t_{k}}\right|_{4}^{2} \\
& =\left|\sum_{k=1}^{m_{n}}\left(x_{0}^{t_{k}}\right)^{-1}\left[x_{0}^{t_{k}} \check{Y}_{t_{k-1}}^{t_{k}}\left(x_{0}^{t_{k}}\right)^{-1}-\int_{t_{k-1}}^{t_{k}} x_{0}^{\tau} d \check{Y}_{0}^{\tau}\left(x_{0}^{\tau}\right)^{-1}\right]\left(x_{0}^{t_{k}}\right)\right|_{4}^{2} \\
& =\sum_{k=1}^{m_{n}}\left|\left(x_{0}^{t_{k}}\right)^{-1}\left[x_{0}^{t_{k}} \check{Y}_{t_{k-1}}^{t_{k}}\left(x_{0}^{t_{k}}\right)^{-1}-\int_{t_{k-1}}^{t_{k}} x_{0}^{\tau} d \check{Y}_{0}^{\tau}\left(x_{0}^{\tau}\right)^{-1}\right]\left(x_{0}^{t_{k}}\right)\right|_{4}^{2} \\
& \leq C_{4}(T) \sum_{k=1}^{m_{n}}\left|x_{0}^{t_{k}} \check{Y}_{t_{k-1}}^{t_{k}}\left(x_{0}^{t_{k}}\right)^{-1}-\int_{t_{k-1}}^{t_{k}} x_{0}^{\tau} d \check{Y}_{0}^{\tau}\left(x_{0}^{\tau}\right)^{-1}\right|_{4}^{2} \\
& =C_{4}(T)\left|\sum_{k=1}^{m_{n}} x_{0}^{t_{k}} \check{Y}_{t_{k-1}}^{t_{k}}\left(x_{0}^{t_{k}}\right)^{-1}-\int_{s}^{t} x_{0}^{\tau} d \check{Y}_{0}^{\tau}\left(x_{0}^{\tau}\right)^{-1}\right|_{4}^{2} .
\end{aligned}
$$


Here $C_{4}(T)$ is some constant, and we essentially use the additivity of the stochastic integral $\int_{s}^{t} x_{0}^{\tau} d \check{Y}_{0}^{\tau}\left(x_{0}^{\tau}\right)^{-1}$ for intervals, which follows from the independence of the limit (19) of the sequence of partitions $\left\{\Delta_{n}[s, t]\right\}$.

Now the right hand side of the expression (39) tends obviously to zero due to (19).

Let us furthermore note that the obvious equality $X_{s}^{t}=\left(x_{0}^{s}\right)^{-1} \bar{X}_{s}^{t} x_{0}^{t}$ follows from the formulas (23) and (24). Using it, the equality (38) and the estimates (8) we obtain the relations

$$
\begin{aligned}
& \left|\sum_{k=1}^{m_{n}}\left(X_{t_{k-1}}^{t_{k}}-x_{t_{k-1}}^{t_{k}}\right)-\check{Y}_{s}^{t}\right|_{4}^{2}=\left|\sum_{k=1}^{m_{n}}\left(X_{t_{k-1}}^{t_{k}}-x_{t_{k-1}}^{t_{k}}-\check{Y}_{t_{k-1}}^{t_{k}}\right)\right|_{4}^{2} \\
& =\sum_{k=1}^{m_{n}}\left|X_{t_{k-1}}^{t_{k}}-x_{t_{k-1}}^{t_{k}}-\check{Y}_{t_{k-1}}^{t_{k}}\right|_{4}^{2} \\
& =\sum_{k=1}^{m_{n}}\left|\left(x_{0}^{t_{k-1}}\right)^{-1} \bar{X}_{t_{k-1}}^{t_{k}} x_{0}^{t_{k}}-x_{t_{k-1}}^{t_{k}}-\int_{t_{k-1}}^{t_{k}}\left(x_{0}^{\tau}\right)^{-1} d \bar{Y}_{0}^{\tau} x_{0}^{\tau}\right|_{4}^{2} \\
& =\sum_{k=1}^{m_{n}} \mid\left(x_{0}^{t_{k-1}}\right)^{-1}\left(\bar{X}_{t_{k-1}}^{t_{k}}-I-\bar{Y}_{t_{k-1}}^{t_{k}}\right) x_{0}^{t_{k}} \\
& +\left(x_{0}^{t_{k-1}}\right)^{-1} \bar{Y}_{t_{k-1}}^{t_{k}} x_{0}^{t_{k}}-\left.\int_{t_{k-1}}^{t_{k}}\left(x_{0}^{\tau}\right)^{-1} d \bar{Y}_{0}^{\tau} x_{0}^{\tau}\right|_{4} ^{2} \\
& \leq 2 C_{5}(T) \sum_{k=1}^{m_{n}}\left|\bar{X}_{t_{k-1}}^{t_{k}}-I-\bar{Y}_{t_{k-1}}^{t_{k}}\right|_{4}^{2} \\
& +2 \sum_{k=1}^{m_{n}}\left|\left(x_{0}^{t_{k-1}}\right)^{-1} \bar{Y}_{t_{k-1}}^{t_{k}} x_{0}^{t_{k}}-\int_{t_{k-1}}^{t_{k}}\left(x_{0}^{\tau}\right)^{-1} d \bar{Y}_{0}^{\tau} x_{0}^{\tau}\right|_{4}^{2} \\
& =2 C_{5}(T)\left|\sum_{k=1}^{m_{n}}\left(\bar{X}_{t_{k-1}}^{t_{k}}-I\right)-\bar{Y}_{s}^{t}\right|_{4}^{2} \\
& +2\left|\sum_{k=1}^{m_{n}}\left(x_{0}^{t_{k-1}}\right)^{-1} \bar{Y}_{t_{k-1}}^{t_{k}} x_{0}^{t_{k}}-\int_{s}^{t}\left(x_{0}^{\tau}\right)^{-1} d \bar{Y}_{0}^{\tau} x_{0}^{\tau}\right|_{4}^{2} .
\end{aligned}
$$

The first term on the right hand side of (40) tends to zero for $\delta_{n} \rightarrow 0$ because of (25) and the theorem from [6]. The second term on the right hand side of (40) tends to zero for $\delta_{n} \rightarrow 0$ by (38), and this limit procedure does not depend on the sequence of partitions $\left\{\Delta_{n}[s, t]\right\}$.

Thus the formula (32) is proved and our theorem is proved, too.

Corollary. Under the conditions of Theorem 2 the formula

$$
X_{s}^{t}=\lim _{\delta_{n} \rightarrow 0} \prod_{k=1}^{m_{n}}\left(\check{Y}_{t_{k-1}}^{t_{k}}+x_{t_{k-1}}^{t_{k}}\right)
$$


is true.

For the proof let us note that the system $X_{s}^{t}$ satisfies all the conditions (4)(6) of Theorem 1. Especially, (32) gives the condition (6). Moreover, the obvious equality $\mathbf{E} X_{s}^{t}=x_{s}^{t}$ follows from the relation (25). Using the formula (31) for $Z_{s}^{t}=X_{s}^{t}$ we then obtain the formula (41).

Remark 3. From Theorem 2 and the formula (31) it follows that the condition on $Z_{s}^{t}$, i.e. the existence of the limit

$$
\check{Y}_{s}^{t}=\lim _{\delta_{n} \rightarrow 0} \sum_{k=1}^{m_{n}}\left(Z_{t_{k-1}}^{t_{k}}-z_{t_{k-1}}^{t_{k}}\right),
$$

is also a sufficient condition for the existence of the limit

$$
X_{s}^{t}=\lim _{\delta_{n} \rightarrow 0} \prod_{k=1}^{m_{n}}\left(\check{Y}_{t_{k-1}}^{t_{k}}+z_{t_{k-1}}^{t_{k}}\right) .
$$

In particular, if $Z_{s}^{t}=X_{s}^{t}$ and if it satisfies the conditions (1)-(4) from [5] then a careful check of the proof of the main theorem in [5] (formulas (14), (15)) shows that for the existence of

$$
\breve{Y}_{s}^{t}=\lim _{\delta_{n} \rightarrow 0} \sum_{k=1}^{m_{n}}\left(X_{t_{k-1}}^{t_{k}}-x_{t_{k-1}}^{t_{k}}\right)
$$

it is sufficient to require the following weaker condition

$$
\sup _{\Delta_{n}[s, t]} \sum_{k=1}^{m_{n}}\left|x_{t_{k-1}}^{t_{k}}-I\right|_{2}^{2}<\infty
$$

instead of the condition (2) in [5]. Thus, due to Theorem 2 the condition (42) will be sufficient for the validity of the formula (41).

\section{References}

[1] Butsan, G.P.: Stochastic semigroups. - Naukova Dumka, Kiev, 1977 (Russian).

[2] Butsan, G.P.: An integral representation of a multiplicative stochastic semigroup without martingality and continuity conditions. - Ukrainian Math. J. 37, 1985, 453-457 (English translation of Ukrain. Math. Zh. 37, 1985, 562-568).

[3] BUTSAN, G.P.: Infinitesimal semigroups for one class of stochastic semigroups. - Ukrainian Math. J. 35, 1983, 189-192 (English translation of Ukrain. Math. Zh. 35, 1983, 221224).

[4] Butsan, G.P.: Primitive semigroups for a class of stochastic semigroups. - Ukrainian Math. J. 35, 1983, 408-411 (English translation of Ukrain. Math. Zh. 35, 1983, 485489). 
[5] BUTSAN, G.P.: Infinitesimal semigroups for stochastic semigroups without continuity and martingality conditions. - Ukrainian Math. J. 37, 1985, 219-225 (English translation of Ukrain. Math. Zh. 37, 1985, 285-294).

[6] Butsan, G.P.: A class of stochastic semigroups. - Ukrainian Math. J. 36, 1984, 1-4 (English translation of Ukrain. Math. Zh. 36, 1984, 3-7).

Academy of Sciences Ukrainian SSR

Institute of Mathematics

Repina 3

$252601 \mathrm{Kiev}$

USSR 